\title{
Effects of Community Based Learning in Ceramic, Sakraloke's Sukhothai
}

\author{
Nirat Soodsang ${ }^{1}$ \\ ${ }^{1}$ Faculty of Architecture, Department of Art and Design, Naresuan University, Thailand \\ Correspondence: Nirat Soodsang, Faculty of Architecture, Naresuan University, Phitsanulok 65000, Thailand. \\ Tel: 66-83-96-2450. E-mail: nirats@nu.ac.th
}

Received: November 4, 2016

doi:10.5539/ass.v13n2p41

\author{
Accepted: December 26, $2016 \quad$ Online Published: January 19, 2017 \\ URL: http://dx.doi.org/10.5539/ass.v13n2p41
}

\begin{abstract}
The purposes of this research were 1) to establish the community-based learning process of Sukhothai Sangkhalok ceramic work; 2) to design Sangkhalok ceramics for marketing competitiveness and community economic development; and 3) to evaluate the learning outcome of student with the community on Sukhothai Sankhalok designs. The research involved a simple-random sample of 40 second year students majoring product and packaging design of the Department of Art and Design. The community-based learning model in Sukhothai Sankhalok ceramic work as a research tool consisted of the learning plan, activities, and evaluation form. The researcher undertook a field work to collect data from the 3 Sangkhalok ceramics manufacturers in Sukhothai province, namely: 1) Suthep Sangkhalok, Tambon Muang Kao, Amphur Muang Suthothai; 2) Usa Sangkhalok Tambon Muang Kao, Amphur Muang Suthothai 3) Bua Sangkhalok, Tambon Muang Kao, Amphur Muang Suthothai. The data analysis focused on 2 areas, i.e. the analysis of ceramics community on its history, community's operation model, and the analysis of students' learning outcome. The analysis employed descriptive statistics and a comparison of learning outcomes against the $80 \%$ criterion with one-sample statistics. The findings suggested that the community-based learning model of Sukhothai Sangkhaloke ceramic work as obtained in this research comprised 4 components: 1) strengthening community economics and community enterprise development; 2) promoting participatory learning process; 3 ) naintaining and building the capacity of local wisdom, and 4) building network and collaboration to link the knowledge and local wisdom. The Sangkhalok ceramic design works were in 3 collections: 1) Sangkhalok ceramics in swan shape with decorating technique of color painting under the glaze, in 4 styles, and 2) Sangkhalok ceramics in dragon shape with decorating technique of gold color water drawing, in 4 styles, and 3) Sangkhalok in Ganesh shape, in 3 styles. Students who designed the ceramics by the concept of Sukhothai ceramic art model showed the learning outcome with average scores at $84.50 \%$ which was higher than the set criterion at 0.05 level of significance.
\end{abstract}

Keywords: Community-based learning, Ceramics, Sukhothai

\section{Introduction}

Community Based Learning (CBL) is a pedagogical approach with a focus on student's learning through a practice in authentic community situation. The integration of community-based instruction aimed to gain access and acceptance from the community. Students should thus understand their role and view of community, and techniques to work with community. Teachers act as mentor to regularly follow up students' progress and doing activities with community, review with students in what they have learned from community and what the community has learned from them, including students' outcomes. In this way, students will acquire analytical thinking and group process skills. The evaluation tools and methods should vary in corresponding to reality and encompassing the learning objectives.

Learning the Sangkhaloke ceramics work to continue and preserve the arts and culture of the nation is essential for their existence along with the nation. Sangkhalok is the term used to refer to ceramics particularly produced in Sukhothai province since the period of Sukhothai-Ayudhaya kingdom or around the $19^{\text {th }}$ B.E. - early $22^{\text {nd }}$ B.E.. The kiln sites were located at the ancient Sawankalok (currently in Sri Satchanalai district) and at the ancient town of Sukhothai in Amphur Muang Sukhothai. It was assumed that the term Sangkhalok came from the word Sawankhalok as the name of the largest main Sangkhalok production site in that period. The following two areas of ancient Sangkhalok kilns had already been surveyed and excavated. 
1. Sukhothai kilns. A number of kilns were found scattered around the area of Jone river, most of which was called Tao Ta Klub or up-draught kiln for producing Sangkhalok ceramics with draw motif under black glaze. The distinctive feature of Sangkhalok ceramics produced from Sukhothai kilns is that the thick white clay water was applied onto the surface before drawing a pattern or glazing because the soil texture was not fine but rough and in grey or black colors. The pieces of ceramics were arranged in vertical stacks in the kilns, each was interposed by a legged plate of sugarcane, hence leaving traces of 5-6 spots on the inner bottom of each item except the one at the top.

2. Sri Satchanalai kilns. A number of kilns were found scattered around the area of Yom river, most of which was called Tao Pratoon or cross-draught kilns. This type of kilns offers higher quality of Sangkhalok ceramics than other kilns. There were a variety of items manufactured from this site which included the green glazed or Celadon, the white glazed, the brown glazed and 2-color glazed, including unglazed stoneware, produced from major kilns at Baan Kaonoi and Baan Payang.

Sangkhalok ceramics were produced for multiple purposes and uses as 1) a supplement in religious ceremonies or belief rituals to support the event such as the joss stick pot or dishware offered to the temple or monks, offering dolls to remove bad luck, and utensils accompanied the burial by the belief for the deceased person to use in the next life; 2) architectural ornaments such as gable-apex, gabled triangle, bralee, roof tiles, railing, entrance giant guards; 3) daily life housewares such as dishware, jar, jug, small case, water pot; and 4) other purposes as toys, e.g., dolls in human and animal figures, cricket cage.

The community-based learning in ceramic work will enable students to apply it in their work in all sectors of student society, become more apprehensive of the real world, understand individual practice toward the society, and appreciate the lifelong learning process. Integrated pedagogy is a combination of all the knowledge of subject contents to offer a holistic learning and enable its application in daily life. By connecting it to the community-based learning, it empowers the learning in which learners are involved in the community, resulting in both their learning from the community and the community's learning alike, as an authentic learning. It focuses on learner-centered approach, allowing their participation in planning meaningful learning activities corresponding to learners' interest. Teachers take role as manager who facilitates the learning through a range of technical methods such as self-study, experiential learning, situated learning, inquiry-based participatory learning, project-based learning, and case study-based learning. The aim is to enable learner's self-learning and realizing the importance of learning so as to attain multi-dimensional development including memory, rationale, appreciation, aesthetics, communication, self-adjustment, and social responsibility consciousness, as desired characteristics of learner.

The integrated pedagogy for community-based learning is characterized by an effort to integrate subject matters by using a range of learning styles as follows. 1) Situated learning focuses on learner's acquired experience and action under authentic social culture and context which requires social interaction with individuals and environment to construct individual's knowledge. 2) Experiential learning focuses on learner's learning through life experience, work experience, and other forms of experiences, informally. With the learners' reflection on experiences, new learning occurs. 3) Project-based learning allows for student's acquisition of hands-on experience, solving problems with scientific methods, inquiring by oneself, developing skills in work planning, analyzing and evaluating performance by oneself. 4) Case study-based pedagogy focuses on the learner's investigation on their area of interest in authentic situation in relation to the real life problem, and apply it in everyday life. In this approach, community members are involved in the learning of learners. Teachers pose questions or encourage learners' collective thinking and solving the community's problems. Environment integrated pedagogy by community-based approach is characterized by a holistic combination without division in parts but aimed to provide a balance and pleasurable learning, and promote self-learning and teamwork. Educators organize a range of activities to activate learning through stories and building relationship network, and merge those aims to provide a complete story. Classroom atmosphere is arranged to encourage learners' thinking, doing, and becoming confident by allowing them to express idea and act toward their classmates and the public. The strategy to promote learning through the environment integrated pedagogy by community-based approach is to create the learner's "sense" of desire to learn, and change of behavior through observing, thinking, and doing. In this way, learners are facilitated to seek knowledge through cognitive process with teacher's companionship. This management of learning allows for learner's observing, listening, posing questions, recording, hypothesizing, finding answers, presenting to group meeting, using research mechanism for learning, and practicing academic writing and reviewing, and importantly, developing competency to connect and integrate for the wholeness of environment and the relation of all in the society. This connection gives rise to students' awareness of role of oneself as an ethical dimension to environment and society. The application of 
integrated pedagogy by community-based approach in the teaching and learning of environment is relevant to the nature of subject that focuses on field practice with environmental problem situation in the community. It provides a number of advantages, for example, with the transfer of knowledge, the learners develop a holistic view of environment and are able to combine knowledge for meaningful learning and applying in the real life, encounter new situations and solve problems effectively. Moreover, it cultivates learners the environmental ethics and enhances their work in other aspects of wisdom art.

\section{Research Objectives}

(1) To establish a community-based learning process in ceramic work.

(2) To design Sangkhalok ceramics for marketing competitiveness and economic development of community.

(3) To evaluate the learning outcome of student and community on Sukhothai Sankhalok ceramics designs.

\section{Research Methodology}

\subsection{Population and Sample}

The research population included 120 students majoring product and packaging designs of the Department of Arts and Designs. The sample was 40 second year students majoring product and packaging designs of the Department of Arts and Designs, selected by simple random sampling.

\subsection{The Research Tools}

The research tools were the community-based learning model in Sukhothai Sangkhalok ceramic work which consisted of the learning plan, activities, and evaluation form.

\subsection{Data Collection Method}

The researcher undertook a field data collection by conducting interviews with the 3 manufacturers of Sangkhalok ceramics in Sukhothai province. The data was summarized in the following tables to show the management model to create Sangkhaloke ceramics. The 3 manufactures as community learning resources were 1) Suthep Sangkhalok, Tambon Muang Kao, Amphur Muang Suthothai; 2) Usa Sangkhalok Tambon Muang Kao, Amphur Muang Suthothai; and 3) Bua Sangkhalok, Tambon Muang Kao, Amphur Muang Suthothai.

Table 1. Data collection procedure

\begin{tabular}{lll}
\hline Schedule & Procedure & Goals \\
\hline Month 1 & 1) Pre-research stage included 2 steps, i.e. selecting & -The community with good relationship, and the \\
& community and building rapport with the community & development of Sangkhalok ceramic work. \\
Months & 2) Research stage involved exploring and analyzing & - Development of at least 10 styles of Sangkhalok \\
$2-8$ & problems with the community, determining the & ceramic design works with value added of new \\
& problems, designing the research and data collection, products for marketing competitiveness. \\
& analyzing data to make conclusions, presenting research & - Establishment of community-based learning \\
Month & result, using a community-based pedagogical strategy. & process in Sangkhalok ceramic work. \\
10 & 3) Post-research stage involved making the action plan & - Establishment of collective learning network for \\
& with the community, implementing, monitoring and the community and educational institution. \\
& evaluating the action plan, and carrying out in cycles, & \\
each of which comprised planning, implementation, & \\
observation, and reflection.
\end{tabular}

\subsection{Data Analysis Methods}

The researcher analyzed into 2 major areas which included the analysis of ceramic community in the aspects of background history, community's operation model; and the analysis of students' learning outcomes. The research took on descriptive statistics and a comparison of learning outcomes based on the set criterion of $80 \%$, using one-sample statistics.

\section{Research Result}

1. The community-based learning process in Sangkhalok ceramic work encompassed 4 elements, namely: 1) strengthening community economics and community enterprise development; 2) promoting participatory learning process; 3) maintaining and building the capacity of local wisdom; and 4) building network and collaboration to link the knowledge and local wisdom. 
1.1 Strengthening community economics and community enterprise development involved the improvement of standard quality of production/product, increasing the productivity of community products/items for their economic value added with better price per unit and lower production cost or raising income and/or lowering expense by introducing activities to develop product styles that meet the market preference.

1.2 Promoting participatory learning process focuses on learning outside the classroom with an aim to enhance skills and competency of community members by collaborating with educational institutions. This is to establish a lifelong learning culture that aligns with the community way of life, and emphasizes on the community as a base for learning that allows for direct and easy access to knowledge, and being capable of thinking and doing. Moreover, it supports a systematic involvement of community throughout the process from problem analysis, making suggestions, examination, demonstration, making decision, acceptance, and evaluation, particularly focusing on mutual practice in every step with common spirit in problem solving and local development. The design of learning activities considered 3 aspects, i.e. standard of learning outcome, pedagogical strategy used for learning development, and strategies for evaluation of learning.

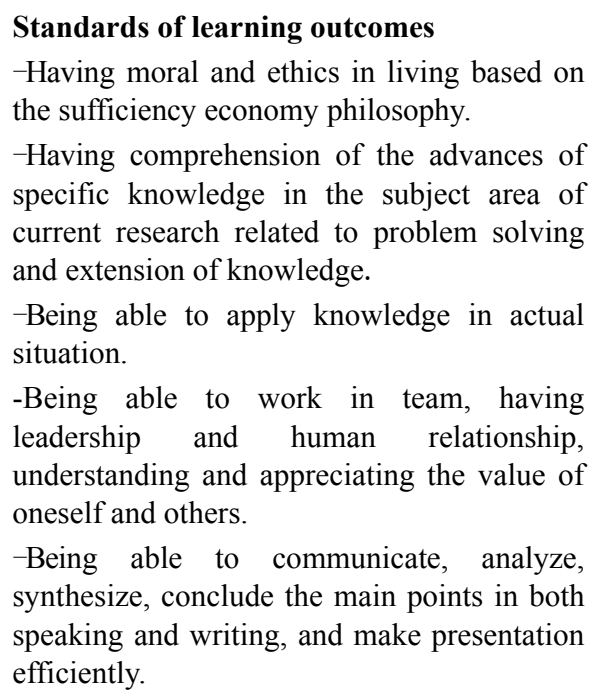

\author{
Pedagogical strategy used for learning development \\ -Project-based learning \\ -Work-integrated learning \\ -Field trips \\ -Professional training / Co-operative education

\section{Strategies for Evaluation of Learning} \\ -Evaluation of knowledge and skills by means of practical \\ examination, oral examination, observation, learning behavior. \\ -Evaluation of learning attitude, using a questionnaire or a self-report. \\ -Evaluation of tasks assigned to students. \\ -Evaluation by professional training sites/establishments
}

1.3 Maintaining and building the capacity of local wisdom in the community focuses on the transfer and utilization of local wisdom to extend research and to process and produce products by investigating and developing a collecting and storing system for a database of knowledge; setting social assurance for local sages such as by supporting fund to maintain and transfer local wisdom, supporting the use of local wisdom as well as the diversity of Thai arts and culture as a way of life, precious value, and Thai-ness, aiming to explore, learn, recover, develop, transfer, and disseminate to the global society.

1.4 Building network and collaboration to link the knowledge and local wisdom, as well as alliances of commercial activities between community and various organizations of both the government and private sectors.

2. The result on Sangkhalok ceramic designs with at least 10 styles for marketing competitiveness and community development

The study result on the ceramic styles produced by Suthep Sangkhaloke, Usa Sangkhaloke, and Bua Sangkhaloke revealed similar characteristics of their operation with 2 types of production. 1) The major products remained traditional style of Sangkhalok ceramics such as basins, dishes, bowls, dolls, etc. but with some pieces of lower or larger size currently depending on the crafter's ability to form the pieces. 2) The product style made to order by customers included such as wall tiles, sculptures, e.g., the Ganesh. The styles of product being developed were partly dependent on customers' preference, and the government agencies also aid in making the designs. However, most was developed by the manufacturers themselves, while maintaining the uniqueness of Sangkhalok ceramics in their shape, pattern, and size.

With respect to the transfer of wisdom, the manufacturers as the business owners had learnt from their parental generations and then convey such knowledge to their offspring and relatives to expand the business and set up factories to earn by themselves. Since the ceramic work is largely a round-relief type that requires crafters' mastery with decades of experience, the tools used were mostly those essentials such as clay extruder, kiln, pot 
mill, while other tools apply local materials. The main source of raw material is Sukhothai, using clay in the local area and mixed with compound clay (commercial finished clay) as an exclusive formula of Suthep Sangkhalok. Suthep Sangkhakol used ash glaze with raw materials ordered from Bangkok and general markets. For manufacturing procedure, the work pieces or products of this factory were made to order for most of them. The crafter will form the work piece using the pre-pressed, plastic clay bar to form the piece with a round-relief method to make a range of items or use the method of wheel throwing, letting dry, and biscuit firing. Next are drawing colors and glazing, then again burning in kiln. There are 2 ways of decorative technique used on these products.

1. The items produced in traditional styles were decorated with color draws under glaze in several ancient patterns such as fish, creeping plants, etc. mainly in black and in other colors like red and green as well, and applied celadon glaze identical to ancient works.

2. The items of round-relief such as the Ganesh and god figures were decorated with stucco by using clay with colors different from the item body, and adding with drawing pattern, followed by either clear or celadon glazing.

The ceramic items of Suthep Sangkhalok were distributed in Thailand and in oversea countries. About $95 \%$ of them were sold to Thai and foreign tourists visiting the ancient city of Sukhothai, and also distributed to those shops at Suan Chatuchak in Chiangmai, and another $5 \%$ were exported to the UK and Germany.

The problem and constraint were related to the lack of basic knowledge on ceramic production, hence with delayed learning and requiring an attempt to succeed. Another problem was less and discontinued support by the government. Finally, the cost problem particularly the expensive gas increased manufacturing cost.

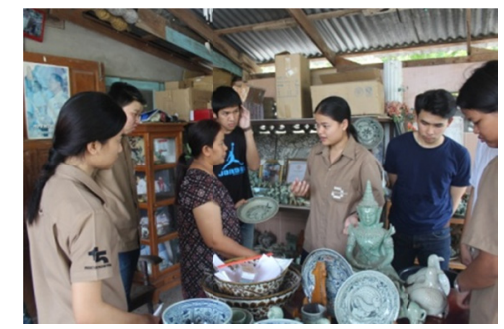

Figure 1. Students entered the community to learn about the production of Sangkhalok ceramics

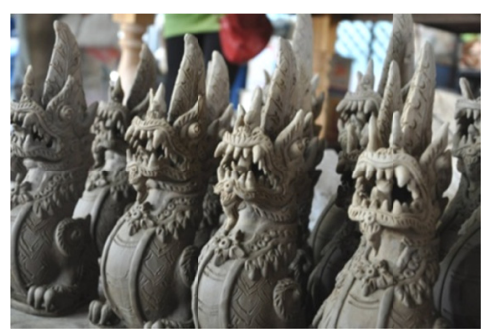

Figure 3. The feature of Sangkhalok in Sing-shape using pressing mold and stucco decoration in production process

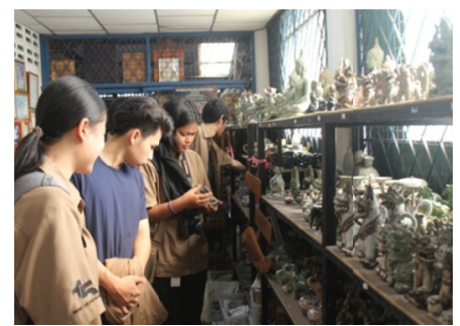

Figure 2. Study on the styles of ceramics sold at Suthep Sangkhalok manufacturer

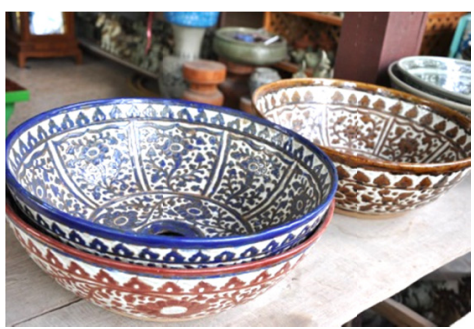

Figure 4. Patterns found in colored drawing decoration dominantly in plants or animal figures

\section{Design works}

The works followed preservative designs, process, unique styles, clay body and patterns. The products of strength clay body or stoneware were burnt in the high temperature at $1150-1280^{\circ} \mathrm{C}$. The decorative techniques and patterns were varied as illustrated below.

Collection 1 Clear glazed ceramics with patterns drawing under dark brown glaze, similar to Chinese and Vietnamese potteries

Collection 2 White glazed ceramics with patterns drawing above golden brown glaze

Collection 3 Kai-Ka green glazed ceramics or Celadon with scratch and chamfer decoration in the clay body, and glaze, similar to Chinese pottery in the late Song Dynasty to Yuan Dynasty (around the $19^{\text {th }}-20^{\text {th }}$ B.E.)

The patterns appeared in Sangkhalok ceramics are very unique and mostly signify different aspects of auspiciousness. For example, the 8 fortunes are represented by Paed Sian pattern. Others include animal patterns, 
phanprueksa (plant) patterns such as peonies representing wealth, beauty; chrysanthemum referring to sustainability; bat signifying happiness and longevity. The unique patterns are those of animals, e.g., fishes usually accompanied with phanprueksa.

Category 1 Clear glazed ceramics with patterns drawing under dark brown glaze, mostly as water container in pitcher shape with long, small neck and swan-like mouth.

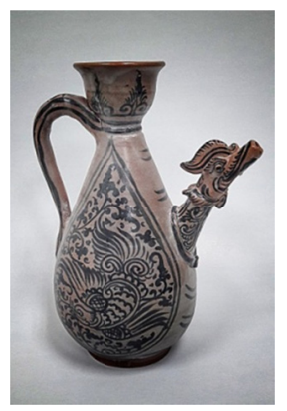

Figure 4. Bird pattern surrounded by flowers and leafs patterns

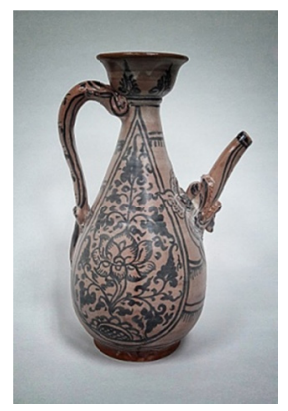

Figure 5. High-shaped pitcher or water jug with lotus pattern

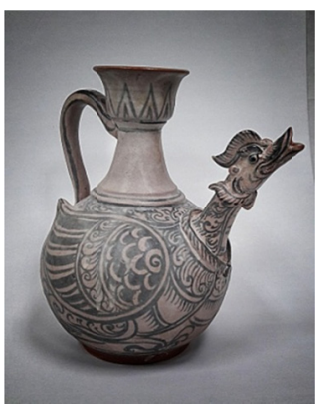

Figure 6. Swan-shaped pitcher, short tail, with color drawing under glaze around the wing and body

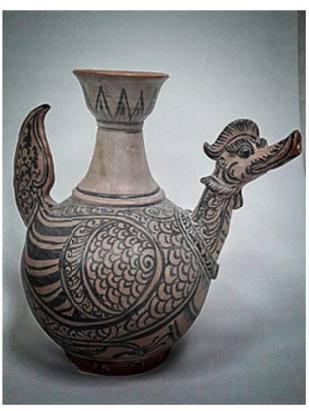

Figure 7. Swan-shaped pitcher, long tail, with color drawing under glaze around the body

Category 2 White glazed ceramics with patterns drawing above golden brown glaze

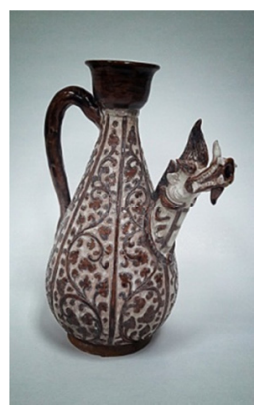

Figure 8. Dragon-shaped pitcher, white glaze and gold color water drawing, with plant pattern

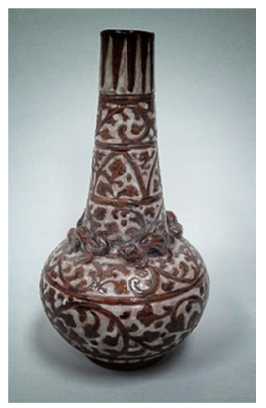

Figure 9. Water jug, white glaze and gold color water drawing

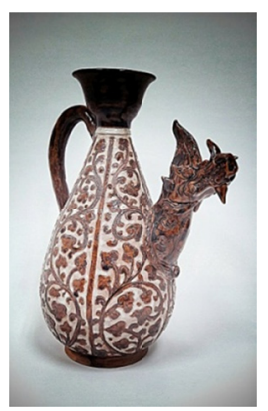

Figure 10. Dragon-shaped pitcher, white glaze and gold color water draw, with plant pattern

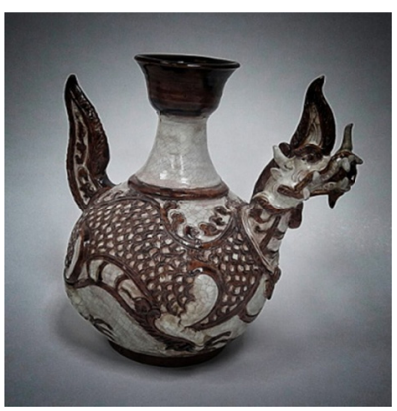

Figure 11. Swan-shape pitcher, long tail, with color drawing under glaze around the body

Category 3 Kai-Ka green glazed ceramics in Ganesh collection of 3 styles (Postures)

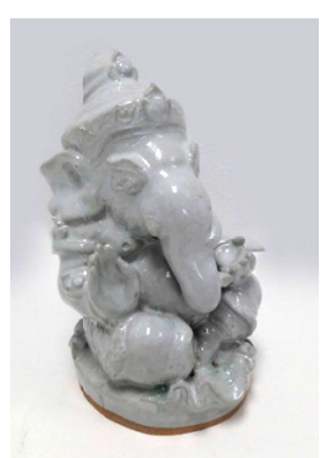

Figure 12. Two-hand-style with a bowl in the left hand

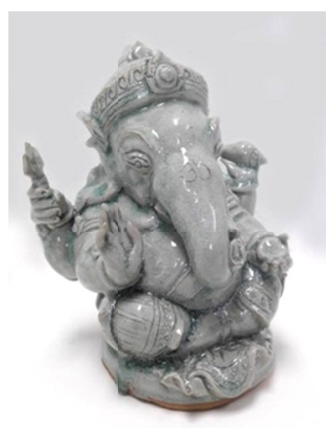

Figure 13. Four-hand-style with a Tri in the right hand, a Sang in the back left hand, and a bowl in the front left hand

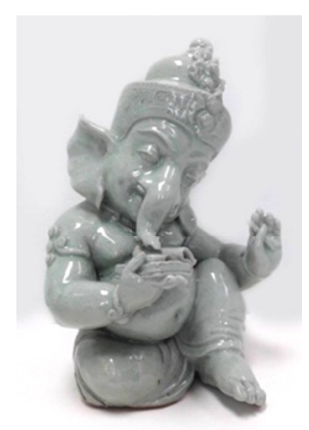

Figure 14. Two-hand-style with a scripture in the right hand and a pen in the left hand 


\section{Evaluation result of students' learning with the community on Sukhothai Sangkhalok ceramic designs}

The result based on the experiment of community-based learning plan in Sukhothai Sangkhalok ceramic work with 40 students in their study visit to learn about the production of ceramics at the 3 manufacturers. The students acquired the learning through observation, inquiry, interviews with the business operators on the current status, problems, and the direction of designs to satisfy the market need. They worked with the community to make the designs and sketches, and had a try to produce ceramic pieces. The learning outcome of students indicated that they can achieve $84.50 \%$ as shown in Table 2 .

Table 2. Result on a comparison of students' learning scores against the $80 \%$ criterion, using one-sample statistics

\begin{tabular}{cccccc}
\hline List & $\mathrm{N}$ & Mean & S.D. & $\mathrm{t}$ & Sig. (2-tailed) \\
\hline Design scores & 40 & 84.50 & .95 & $2.231^{*}$ & .031 \\
\hline
\end{tabular}

$\mathrm{P}^{*}<0.05$ Significant difference from the criterion at the level of 0.05

Table 1 illustrated students' having mean scores, i.e. $84.50 \%$, above the set criterion on the ceramic designs by the concept of Sukhothai ceramic arts.

\section{Discussion}

According to the research result, the community-based learning process in Sukhothai Sangkhalok ceramic work comprised 4 elements, i.e. 1) strengthening community economics and community enterprise development; 2) promoting participatory learning process; 3 ) maintaining and building the capacity of local wisdom; and 4) building network and collaboration to link the knowledge and local wisdom. Relevant findings were found in Bduahakosa (2011) and Buajoom, Intarakamhang, Boonpraokob, and Thanachanan (2014) in their study to develop a learning model by community based approach for establishing both vocational interests and skills on local careers for the students. Their study aimed to develop local curriculum on the learning substance group of career and technology under the community based approach; study the results of change in the interests and skills on the local careers under the community based approach; and develop a learning model by community based approach. It included 21 participants, i.e. school principal, teachers, parents, school committees, the local sages, and 23 students, totaling 44 participants. It involved 2 phrases: phrase 1 for the preparation of participants, and phrase 2 for planning, implementation, and improvement for student's interests and skills in local career. Both quantitative and qualitative approaches were employed for data collection. Quantitative tools were a questionnaire on interests, knowledge, and attitudes on the careers of weaving in Luk Kaew pattern and making weaving equipment; and a check-list observation forms for assessment of working skills in both careers. Qualitative tools were in-depth interviews and participatory observation. The research findings were as follow: 1) The result provided a 60-hour local curriculum development on the weaving in Luk Kaew pattern and making weaving equipment through the learning from the local sages along with on the job training. The 5 steps of local curriculum development were (1) analyzing the problems and students expectation, (2) determining the career in the local area, (3) creating the local curriculums, (4) reviewing the local curriculums, and (5) testing and improving the curriculum. 2) The result of change in the interests and skills in local careers showed that (1) students learning weaving Luk Kaew pattern displayed high level of interest which was higher than the determined standard, and those learning to make weaving tools had their interest by the determined standard; and (2) students in both curriculums showed good skill level in local career. 3) The learning model under the community based approach consisted of 7 steps: (1) studying the community's capacity, (2) encouraging and empowering both local people and school staff to develop curriculums together, (3) studying the local career development needs of students, (4) creating local curriculums, (5) managing learning activities focusing on hands-on training, (6) conducting learning-aided projects, and (7) evaluating the authentic learning results. Another study was conducted by Siriphantana and Uamcharoen (2014) on the development of community-based program on Thai folklore in higher education with the purposes to develop the community-based program on Thai folklore in higher education; experiment the community-based program; and study the efficiency of the program. The population was Nakhonpathom Rajabhat University and the sample was the second year students of Thai Education major in 2014 academic year, comprising 3 groups and 1 volunteers group, totally 35 participants. The sample was purposively selected. The instruction was performed for 16 weeks, four periods per week. The research instruments included Thai folklore course (TQF3), 12 instruction management plans, a handbook of community study activities, a folklore learning outcome test, and a questionnaire on students' opinions toward the instruction. Data analysis employed t-test, frequency, percentage, mean, standard deviation, 
and content analysis. They found the followings. 1) The development of community-based program with a focus on teachers and learners consisted 4 steps, i.e. Plan: P, Instruction: I, Implementation: I, and Evaluation: E. 2) The mean scores were found higher at 0.1 significance level at post-learning than at pre-learning. 3) The students' opinions towards the program were at high level, that is, the students learnt from surroundings.

The design works of Sangkhalok ceramics in this research were obtained in 3 collections, namely: 1) Sangkhalok ceramics in swan shape with decorating technique of color painting under the glaze, in 4 styles, and 2) Sangkhalok ceramics in dragon shape with decorating technique of gold color water drawing, in 4 styles, and 3) Sangkhalok in Ganesh shape, in 3 styles. The result on students' learning base on mean scores of their ceramic designs by the concept of Sukhothai ceramic art model was at $84.50 \%$ which was higher than the set criterion at 0.05 level of significance. The finding agreed to Sivaleeravilas (2006) in her study on the development of community-based education models for social capital development that aimed to analyze the community-based education models for social capital development in successful communities; to develop the community-based education models for social capital development in the community in crisis; and to propose the developed community-based education models for social capital development. The researcher adopted the structural-functionalism theory as the conceptual framework and used a qualitative approach for field research in 3 communities. The data were collected by means of focus-group discussion, in-depth interview, participatory and non-participatory observation. The proposed community-based education models for social capital were examined by specialists and members of the community in crisis. The result was as follows. 1. The community-based education models for social capital development comprised informal, formal, and non-formal education as mutual learning among community members. Informal education was found to be most important since it was an every-day life learning process empowered by potentialities and participation of the members of the community. The main function of informal education was to carry on the value system, spirit, and local wisdom that allowed for further application and extension in the present context. The formal and non-formal education served to empower community's learning in an attempt to establish common value and appreciation of value, spirit, and local wisdom. 2 . The education model for social capital development in the community in crisis could be applied in those communities with insufficient knowledge and learning resources as the knowledge base. In such community, an emphasis has to be put on techniques that encourage learning for social capital development by focusing on setting an exchange forum for collective learning among community members and their search for value, spirit, and local wisdom so that these can be carried on and applied in the social capital in current context.

\section{References}

Bduahakosa, K. (2011). A Handbook for the Development of Learning Community. Bangkok. Office of the National Research Council of Thailand.

Buajoom, D., Intarakamhang, U., Boonpraokob, P., \& Thanachanan, S. (2014). The Development of Learning Model by Community Based Approach for Establishing both Vocational Interests and Skills on Local Careers for the Students of Ban-Panaeg Wittaya School. Journal of Behavioral Science, 20(2).

Siriphantana, C., \& Uamcharoen. (2014). Development of Community-based Program on Thai Folklore in Higher Education. Silpakorn Educational Research Journal, 8(1).

Sivaleeravilas, P. (2006). Development of Community-based Education Models for Social Capital Development. Retrieved November 19, 2015, from http://www.researchgate.net/publication/46141079_

\section{Copyrights}

Copyright for this article is retained by the author(s), with first publication rights granted to the journal.

This is an open-access article distributed under the terms and conditions of the Creative Commons Attribution license (http://creativecommons.org/licenses/by/4.0/). 